\title{
Los sistemas productivos tradicionales y el programa RESA en el resguardo Ticoya de Puerto Nariño
}

Traditional production systems and the RESA program at Ticoya guard in Puerto Nariño

\author{
Juan José Vieco \\ Ph. D. en Antropología. \\ Profesor asociado, Departamento de Antropología \\ Universidad Nacional de Colombia, Sede Bogotá. \\ Contacto: jjviecoa@unal.edu.co
}

Artículo de investigación

Fecha de recepción: febrero 25 de 2015 • Fecha de aprobación: mayo 6 de 2015

\section{RESUMEN}

El presente artículo es el resultado de una investigación realizada en el resguardo Ticoya, del municipio de Puerto Nariño, localizado en la Amazonia colombiana, donde a través de programas asistencialistas de desarrollo impulsados por el estado colombiano, se trata de mejorar la situación alimentaria de los pueblos indígenas y campesinos del país. El programa RESA, Red de Seguridad Alimentaria, parte del principio de que las comunidades objeto de este se encuentran en estado de desnutrición debido a la pobreza de sus sistemas productivos y a la falta de generación de ingresos económicos que complementen su dieta alimenticia. En últimas se ve a estas comunidades como producto del subdesarrollo, de sus condiciones de atraso y a la inexistencia de ciencia y tecnología modernas.

Palabras clave: Amazonia, Resguardo Ticoya, Sistemas productivos tradicionales, seguridad alimentaria, conocimiento local, sistemas híbridos. 


\begin{abstract}
This article is the result of a research conducted in the Ticoya shelter, in the municipality of Puerto Nariño, located in the Colombian Amazon, where through assistance development programs promoted by the Colombian state, the food situation is improving indigenous peoples and peasants in the country. The RESA Food Security Network program is based on the principle that communities object of this welfare program, are in a state of malnutrition because of the poverty of their production systems and the lack of income generation to supplement their diet. At last, these communities are seen as a product of underdevelopment, their conditions of backwardness and the lack of modern science and technology.
\end{abstract}

Keywords: Amazon, Ticoya guard, traditional production systems, food security, local knowledge, hybrid systems.

\title{
INTRODUCCIÓN
}

La principal característica de los planes de desarrollo impulsados por el Estado en los territorios indígenas, es el fomento de la generación de ingresos económicos mediante la integración creciente al mercado. De esta manera, se cumplirá con el propósito de "elevar la calidad de vida", objetivo que figura en todos los planes de desarrollo estatales. Esta concepción lleva implícita una visión particular del desarrollo, que en última instancia es la forma en que el Estado colombiano entiende cómo debe establecer su relación con los pueblos indígenas.

De esta manera, la ciencia y la tecnología se convierten en un aspecto fundamental de los proyectos estatales de desarrollo, de las organizaciones no gubernamentales (ONG) y de los planes de vida. En los primeros, su aplicación está relacionada con el crecimiento económico pues el objetivo de los planes, en el campo de la producción, es generar ingresos económicos, objetivo relacionado con propósitos a largo y mediano plazo que reiteradamente figuran en dichos proyectos: mejorar la calidad de vida de las comunidades indígenas. Así, en la práctica se reproducen con una diferencia de más de sesenta años, los preceptos invocados por el presidente Truman: los problemas del desarrollo se solucionan con la ciencia, la tecnología y el conocimiento moderno (Viola, 2000). El problema de la pobreza y la marginalidad no responde, entonces, 
a la desigualdad en la distribución del ingreso, factor inherente a la lógica misma del capitalismo, sino a las condiciones de atraso de los pueblos indígenas, producto de un conocimiento pre moderno - pre lógico lo llamaría Lévy-Bruhl- y a la inexistencia de ciencia y tecnologías modernas.

Los proyectos de desarrollo, que responden más a objetivos asistenciales y que no fomentan el desarrollo propio, son impuestos desde los organismos centrales del Estado, pretendiendo resolver de este modo la falta de planeación local, la cobertura y la escasez de recursos de inversión, pues en la resolución de los problemas sociales del país se aplican también los conceptos de economía de escala: lo que funciona en un lugar debe hacerlo en todas partes. Las cooperativas diseñadas para campesinos caficultores de ladera deben funcionar en los pueblos indígenas del Amazonas, dejando de lado así, desde el comienzo, la posibilidad de abrir espacios a concepciones y objetivos distintos a los definidos previamente como "necesidades y carencias".

Teniendo en cuenta el contexto en el que generalmente se han puesto en práctica los proyectos de desarrollo, a continuación se analizará el caso del programa Red de Seguridad Alimentaria (RESA) ejecutado entre 2004 y 2007, como su nombre lo indica se dirige al fortalecimiento de los sistemas productivos tradicionales y modernos; su filosofía se expresa con su lema "producir para no comprar lo que la tierra nos puede dar” (Red de Solidaridad, informe final RESA, 2006, p. 2). Este también buscaba incentivar una cultura del ahorro y estimular responsabilidades individuales frente a los proyectos productivos para garantizar el éxito del programa.

\section{El programa Red de Seguridad Alimentaria (RESA) de Puerto Nariño}

El programa RESA fue formulado por el primer gobierno de Álvaro Uribe (20022006) con el fin de mitigar la crítica situación de alimentación de las comunidades campesinas e indígenas del país. La situación de inseguridad, del violento conflicto armado y el desplazamiento forzado, había agudizado el problema de la seguridad 
alimentaria $^{1}$ que existía en estas comunidades debido a factores como la dependencia del mercado de sus sistemas productivos y a las desigualdades sociales, económicas y políticas generadas por el sistema capitalista.

El programa RESA se enmarcó en la política estatal del Plan Nacional de Seguridad Alimentaria y Nutricional (PNSAN). De este plan se derivó el plan de alimentación formulado por organismos internacionales como el Banco Mundial, la FAO, centros de investigación y universidades de países desarrollados. Bajo esta óptica, la alimentación y la nutrición se constituyen como un objeto científico, como un conocimiento de expertos, a comienzos de los años setenta, que dan forma a políticas de planificación de la alimentación y nutrición $(\mathrm{FNPP})^{2}$ y que se implementan a partir de los ańos ochenta en los países del Tercer Mundo (Escobar, 1998, p. 204). De esta manera, mediante el conocimiento experto se construyen las relaciones de poder de las instituciones y las comunidades locales, al tiempo que se invisibilizan y omiten los conocimientos locales.

La desnutrición y el hambre han sido objeto de discursos y prácticas del desarrollo, el cual ha moldeado y tratado el problema de la alimentación como un problema aislado de las condiciones históricas de los pueblos y sectores de población a los cuales se dirigen estos programas. Los programas como RESA basan sus objetivos en decisiones racionales y hacen uso de técnicas científicas, manejadas por expertos, que formulan y dirigen estos programas tales como las encuestas, predicciones, análisis de la situación encontrada antes de la ejecución del programa y el análisis costo-beneficio.

Acorde con los preceptos que orientan los proyectos de desarrollo implementados por el Estado, el programa RESA se rigió por principios de racionalidad económica, derivados de la escuela económica clásica y neoclásica, entendidos

1 El concepto de seguridad alimentaria, acuñado por la organización de la Naciones Unidas para la Agricultura y la Alimentación (FAO), ha sido cuestionado por organizaciones de base como Vía Campesina y en su lugar proponen el concepto de soberanía alimentaria, cuyo énfasis se centra en los conocimientos productivos propios de las comunidades locales para la provisión de alimentos y como una alternativa frente a la dependencia de las multinacionales de alimentos.

2 FNPP, Food and Nutrition Policy and Planning. 
como la asignación óptima de recursos a planes de desarrollo de alimentación, en este caso del resguardo Ticoya de Puerto Nariño, con el fin de superar los problemas de desnutrición detectados por las agencias gubernamentales como el Instituto Colombiano de Bienestar Familiar (ICBF). En efecto, de acuerdo con esta entidad, en los últimos años entre el 28 y el $31 \%$ de la población del Trapecio Amazónico sufre de desnutrición crónica, lo que representa casi el triple del promedio nacional. La desnutrición aguda se sitúa en el $6.6 \%$, seis veces más que el promedio nacional, que se ubica en el 0,8 \% (RESA, 2006, p. 22).

RESA es un claro ejemplo de un programa concebido de arriba hacia abajo (topdown), desde las oficinas del centro del país para ser aplicado en todas las regiones bajo los preceptos de la economía de escala, con el fin de lograr mayor cobertura con menos recursos. Las características culturales de los pueblos indígenas no se tuvieron en cuenta para la aplicación del programa RESA, pues asumió que los sistemas productivos indígenas y sus patrones de alimentación, coinciden con las características de los sistemas agrarios y campesinos del país. El programa RESA se basó en tres componentes básicos: motivación, difusión e insumos. Su filosofía expresa lo siguiente:

RESA, antes que un programa tecnológico, es un programa sociocultural de economía rural, que pretende "cambiar la actitud" de los pequeños productores rurales frente al uso de la tierra, impulsándolos a sembrar para no comprar los productos alimenticios que la tierra pueda producir (Universidad Javeriana, 2007, p. 4).

Aunque la filosofía del programa tuvo como fin disminuir la dependencia del abastecimiento externo a través del mercado, el problema de la seguridad alimentaria se redujo a la aplicación de unos recursos e implementación de unas medidas para racionalizar los problemas de deficiencia de los sistemas productivos indígenas. En efecto, el programa RESA se dedicó a repartir herramientas, algunas semillas y dictar cursos de capacitación, como se evidenciará más adelante.

Los objetivos del programa RESA se definieron como una política nacional, dirigida a atenuar el avance de la pobreza generalizada en el país que en la actualidad se ubica 
en el $70 \%$ de la población colombiana, en especial en las áreas rurales. Sus objetivos estaban dirigidos a aliviar los problemas de desnutrición y desabastecimiento de poblaciones víctimas del desplazamiento, la violencia y el conflicto armado.

Para el caso de Puerto Nariño, donde la situación de desplazamiento, conflicto y violencia no reviste las características críticas del interior del país, el programa RESA intentó modificar los objetivos del nivel nacional, adaptándolos a las condiciones regionales, en especial a los problemas derivados de los cambios sufridos por los sistemas productivos indígenas y sus consecuencias en los niveles de nutrición y alimentación adecuadas de los habitantes del resguardo Ticoya. No obstante, el marco de maniobra para ajustar el programa a las condiciones específicas de las comunidades del resguardo Ticoya, fue muy limitado. Al respecto, un funcionario del Instituto Amazónico de Investigaciones científicas (Sinchi), entidad encargada de la coordinación e implementación del Programa RESA del resguardo Ticoya de Puerto Nariño, anotó lo siguiente:

El programa RESA de Puerto Nariño se realiza con base en una formulación genérica, si usted me lo perdona, en el sentido de que debía sustentarse en el marco de las directrices nacionales de RESA entonces no podía salirse de ahí sino en el marco de esas directrices, y exactamente en la línea con las temáticas que manejaba RESA a nivel nacional, entonces obviamente tenía unas particularidades en términos de la formulación, de la población, en el caso de los pueblos indígenas, en lo que tiene que ver con el área, bueno eso. Pero más allá no, todo, digamos era, en cierta manera como muy estándar si me perdonas esa formulación, o sea que la posibilidad de digamos si me preguntas cómo fue la intervención del Sinchi ahí, fue más de darle una ayuda a Acción Social, en apoyarlo, en ejecutar ese proyecto en el marco de la experiencia que ha tenido el instituto Sinchi con pueblos indígenas (entrevista del autor, 2012).

Los objetivos trazados para el programa RESA en las comunidades del resguardo Ticoya de Puerto Nariño fueron los siguientes: 
1. Dotar a las familias que integran las comunidades indígenas de herramientas, semillas, pie de cría para afianzar el sector agropecuario y fortalecer la actitud del indígena de producir para comer y no para vender.

2. Brindar la asistencia técnica, talleres de capacitación, visitas de seguimiento, acompańamiento de los procesos y gestión comunitaria a través de las instituciones participantes del proyecto y el equipo de profesionales que destine el comité técnico del proyecto con el fin de mejorar el nivel de seguridad alimentaria a los procesos productivos tradicionales, y el arraigo de su región.

3. Diagnosticar, como parte de la asistencia técnica, en las comunidades beneficiadas el estado de uso del recurso suelo, el nivel de fertilidad de los suelos de las chagras y su relación con el número y estado de plantas cultivadas.

4. Capacitar a las familias para que reconozcan y entiendan el problema de baja fertilidad de los suelos y qué prácticas e insumos de bajo costo pueden implementar para mejorar la productividad de sus chagras y el nivel de seguridad alimentaria de sus familias.

5. Diagnosticar a nivel familiar, la calidad de dieta que tienen durante el ańo, identificando la procedencia de productos, y su relación con el estado de nutrición y salud familiar.

6. Identificar la necesidad y expectativas de las comunidades con respecto a la zoocría o levante de especies menores para el complemento proteico en su dieta alimentaria (Acosta, Peña y Mazorra, 2006, p. 6).

Para cumplir con estos objetivos, el programa selecciona las comunidades y el número de familias beneficiarias.

El proyecto cubrió a 423 familias de 14 comunidades indígenas del Resguardo Ticuna, Cocama, Yagua de Puerto Nariño, según la tabla (RESA, 2006, pp. 6-8). 
La funcionaria de Acción Social describió los objetivos del programa RESA de la siguiente manera:

Básicamente RESA es un programa de seguridad alimentaria que tiene establecido Acción Social. Son dos objetivos: una parte de motivación, donde las familias reciben capacitaciones, material de promoción y divulgación, para que ellos puedan entender el tema de la seguridad alimentaria, sin tener que depender del exterior, sino que ellos mismos la puedan producir en sus chagras o en el caso de otros municipios en el campo, que no tenga ninguna dependencia, ojalá con el mundo exterior, como que ellos mismos se abastezcan. Entonces es una etapa de motivación y divulgación y la otra etapa de entrega de insumos para que puedan desarrollar la actividad de mejorar la chagra, de crear una granja, lo que se elija en la etapa de motivación (Entrevista 02, funcionaria de Acción Social, septiembre de 2009).

Aunque en los objetivos trazados por el programa RESA se pretendió reconocer la importancia de los sistemas productivos indígenas y establecer las condiciones para lo que se podría denominar un diálogo de saberes, entre el programa como proyecto de desarrollo comunitario y los sistemas productivos indígenas, RESA se redujo a ser un proyecto asistencialista del Estado.

En efecto, el conocimiento local se ignoró, pues la forma en que se formularon los objetivos, parecía estar dirigida hacia una comunidad desprovista de cualquier conocimiento local sobre su medio y sus sistemas productivos. De esta manera se estableció un diálogo de una sola vía, desde las instituciones gubernamentales hacia la comunidad.

Para el programa RESA en Puerto Nariño, el problema de la seguridad alimentaria se planteó desde una categoría relacional de separación entre lo tradicional y lo moderno, donde lo tradicional se concibe como una situación ideal existente antes del contacto con la cultura occidental, en la que la alimentación era abundante y equilibrada, mientras que lo moderno se identifica con el cambio social por el que atraviesan los pueblos indígenas del resguardo Ticoya y con la pérdida del conocimiento tradicional de sus sistemas productivos, así como con la adopción de 
modelos de producción insostenibles de acuerdo con las condiciones agroecológicas de la región (Acosta, Peña y Mazorra, 2006).

La autosuficiencia tradicional se confunde con la autarquía total, ya que se considera que los sistemas productivos eran autárquicos e independientes en donde el intercambio estaba restringido a los grupos domésticos, o por completo ausente.

Los sistemas productivos tradicionales se toman como racionales en sí. Sin embargo, se piensa, debido al contacto con sociedades externas y a los cambios que se generan en sus sistemas productivos orientados al mercado, se pierde paulatinamente su condición de racional y en cambio se conforma un sistema productivo irracional, que degrada los suelos y empobrece las chagras.

El programa RESA, no tuvo en cuenta que, más que una separación artificial entre los sistemas productivos entre lo tradicional y lo moderno, lo indispensable es tener en cuenta que los sistemas productivos indígenas son dinámicos y que a través de su relación con la modernidad se realizan intercambios, o en términos de Gudeman y Rivera:

Cómo los sistemas tradicionales entablan "conversaciones" con los sistemas dominantes y muestran cómo, para el modelo de los sistemas productivos locales en Colombia, lo importante es entender cómo este modelo es "el resultado de una extensa conversación... que ocurrió durante miles de años y sigue ocurriendo en muchas tierras" (1990)

Escobar afirma que "estas conversaciones son incorporadas dentro de los modelos locales, produciendo un modelo local de economía” (p. 189). “...los modelos locales no existen en estado puro, sino en complejas hibridaciones con los modelos dominantes" (p. 188).

De esta forma los procesos de cambio ocurridos en los sistemas productivos tradicionales, generados a partir del contacto con los sistemas modernos y el mercado, no se pueden concebir, como lo entendió el programa RESA, como un proceso de pérdida paulatina del conocimiento local del uso y manejo de los recursos naturales 
como el suelo, sino que los sistemas productivos actuales deben ser entendidos como sistemas híbridos, y en cuyo interior se generan tensiones debido al encuentro entre lo tradicional y lo moderno. En este sentido los procesos de cambio no pueden ser comprendidos como un proceso de aculturación, en el sentido de una pérdida continua de valores culturales y la incorporación de unos nuevos, sin que exista la menor relación entre unos y otros.

Las tensiones entre lo tradicional y lo moderno que se experimentan al interior de los sistemas productivos indígenas, reflejan cómo los problemas que buscaba enfrentar RESA, de los suelos, la pérdida de la diversidad de las especies cultivadas en la chagra, la adopción de decisiones de producir productos de la chagra para el mercado, son propios de la dinámica de incorporación de nuevos productos al interior de sus sistemas productivos. Pero esto no significa que el conocimiento local de uso y manejo de sus recursos naturales haya desaparecido y caído en el olvido. Por el contrario, la incorporación de nuevos productos o la intensificación de algunas especies al interior de la chagra, como es el caso de la yuca y el plátano para destinarlos a la venta en el mercado, hace parte de esas "conversaciones". El conocimiento local es un conocimiento práctico, basado en métodos de "ensayo y error" y que se renueva constantemente. Es un conocimiento dinámico, acumulativo y transmitido oralmente de generación en generación. Una de sus principales características es la de innovar, utilizar e integrar los conocimientos externos y adaptarlos a las necesidades locales (Grenier, 1998). Con la llegada de la modernidad no se puede tomar al conocimiento local como un actor pasivo, sometido y reducido a la condición de subalterno de los conocimientos científicos de la cultura de Occidente.

\section{Las comunidades beneficiarias del programa RESA de Puerto Narińo. Su visión y perspectiva del programa frente al desarrollo propio}

RESA fue un programa organizado de acuerdo con una visión moderna del desarrollo, de un discurso construido desde los principios de la economía liberal, que concibe el problema fundamental de cualquier sociedad, moderna y pre-moderna, como lucha incesante por satisfacer unas necesidades ilimitadas de consumo, frente a unos recursos siempre escasos. El objetivo de la economía del desarrollo es lograr un 
equilibrio entre la variable consumo-recurso y la mejor manera de administrarla, de forma tal que permita el equilibrio y la reproducción de la sociedad.

En ese sentido, la economía del desarrollo entiende el problema de la pobreza y de la nutrición, como un campo apropiado para la ejecución de programas de desarrollo, basados, como en el caso del programa RESA, en determinar cuáles son las mejores opciones técnicas y científicas de intervención para solucionar el problema de la desnutrición, cuya causa se atribuyó a los sistemas productivos indígenas, debido a que son insuficientes para suministrar los alimentos necesarios para una alimentación sana y variada capaz de proporcionar un estado adecuado de nutrición.

Investigaciones realizadas sobre nutrición y alimentación entre pueblos indígenas amazónicos por Dufour (1990) y León (2009) entre los Ticuna, establecen que la variedad de cultivos de las chagras indígenas, complementada con actividades de caza, pesca y recolección, es plenamente suficiente para proporcionar una alimentación adecuada y equilibrada. No sobra recordar que los estudios sobre nutrición siguen los parámetros de peso y talla fijados bajo estándares internacionales, los cuales no tienen en cuenta las características particulares de los pueblos indígenas, por lo que se considera que se encuentran en estado de desnutrición.

Varios autores que han abordado el estudio de los sistemas productivos Ticuna, han establecido la diversidad de cultivos, mediante el aprovechamiento integral de los diversos ambientes amazónicos. Los Ticuna poseen chagras tanto en la várzea, como la tierra firme y mantienen huertos habitacionales en los alrededores de sus casas. Los cultivos de la várzea se favorecen por la inundación anual del río Amazonas, que aporta limo y nutrientes a los suelos. En este ambiente las especies cultivadas deben ser de ciclo corto, debido al riesgo de una súbita inundación. Los Ticuna han desarrollado variedades de yuca de ciclo vegetativo corto, entre 4 y 7 meses, con grandes rendimientos (Acosta y Mazorra 2005, p. 50), mientras en tierra firme el ciclo vegetativo es de nueve meses. La tierra firme está determinada a las áreas que no se ven sometidas a la inundación, pero la calidad de los suelos es menor, pues son suelos arcillosos y de alta acidez. Además explotan los rastrojos (tierras en barbecho o descanso) durante largos periodos, es decir mantienen técnicas agroforestales de aprovechamiento de los recursos naturales. Estas actividades las complementan 
con la caza, la pesca y la recolección, que en su conjunto constituyen el sistema de producción Ticuna.

La diversidad de cultivos en los distintos ambientes (várzea, tierra firme, rastrojos) ha sido caracterizada por varios autores. Nimuendajú (1952) establece que son cuatro los cultivos de los cuales dependen los Ticuna para su subsistencia: yuca brava (Manihot utitissima), yuca dulce (Manihot aipi), maíz (Zea mays) y ñame (Dioscorea sp.); además reconoce otros 36 cultivos diferentes. Glenboski (1977), en su estudio sobre la etnobotánica Ticuna, registra más de 60 especies diferentes, incluyendo más de treinta variedades de yuca. León (2009), encontró 52 especies diferentes en las chagras Ticuna, así como 39 variedades de yuca y 16 de plátano, las cuales distinguen perfectamente de acuerdo con su sabor (2009, p. 93). Goulard (2009) registró alrededor de 33 especies diferentes en las chagras del interfluvio. Acosta y Mazorra (2005) en un estudio realizado sobre la técnica tradicional Ticuna de enterramiento de yuca, reconocieron 39 especies diferentes de yuca, 22 especies de yuca dulce y 17 de yuca brava ${ }^{3}$. Trujillo (2008) en su estudio sobre los ingresos en los hogares indígenas, reporta 51 especies diferentes en las chagras en las comunidades indígenas de los ríos Amazonas y Loretoyacu, 43 en las chagras, 35 en el solar (huerto habitacional) y 23 en los rastrojos. Por su parte, el informe final del programa RESA (2006), halló 54 especies diferentes sembradas en las chagras del resguardo Ticoya de Puerto Nariño y resalta que solo se siembran alrededor de 16 cultivares.

Las comunidades indígenas del resguardo Ticoya entienden las propuestas asistencialistas del Estado, por ejemplo el programa RESA, como una intervención motivada que ellos no han demandado y de la cual ni siquiera han sido partícipes con mínima consulta. La propuesta asistencialista de RESA no es por supuesto la primera intervención de Estado en la región del Atacuari y Puerto Narińo. Cuando se define la soberanía colombiana en el Trapecio Amazónico, tras el conflicto con el Perú en 1932, comienza el proceso de conformación de los actuales asentamientos humanos localizados en la ribera colombiana del río Amazonas y sus afluentes que en la actualidad están organizados como resguardos indígenas. El Estado y las entidades

3 Al respecto tanto Nimuendajú (1952) como Goulard (2009) afirman que la yuca brava era desconocida por los Ticuna y su introducción así como la elaboración de la fariña son de fecha reciente. 
gubernamentales, impulsan la creación de asentamientos sobre la base de la oferta de servicios públicos, de salud y educación pero también de infraestructura como la energía eléctrica.

Las comunidades que fueron beneficiarias del programa RESA y en general todas las comunidades del resguardo Ticoya, tienen muy claro el tipo de relación que deben establecer con el "proyecto" y por lo tanto con el Estado y sus instituciones. Saben de antemano que los proyectos solo duran mientras existan recursos y funcionarios públicos al frente de las tareas administrativas, operativas y financieras del mismo. Saben que por un tiempo, (la duración del proyecto), van a contar con ayudas financieras y materiales, con "cosas". O como lo expresa un habitante de la comunidad de Boyahuazú, "se mejoró la calidad de nutrición por un tiempo, más que todo por las semillas".

Los objetivos de los programas de desarrollo asistencialistas del Estado nunca consiguen lo que enuncian en la formulación del programa: la autogestión y apropiación del mismo por parte de las comunidades beneficiarias. En efecto, los proyectos asistencialistas del Estado como RESA, consideran que mediante el efecto demostrativo y a través de la dotación de semillas, herramientas y la realización de talleres de capacitación en la elaboración de abonos orgánicos y concentrado para aves, fundamentado en la superioridad técnica y científica moderna, es suficiente para que las comunidades beneficiarias del programa acojan y se apropien de las enseñanzas de este último.

En ese sentido el programa RESA se estructuró sobre la base de tres componentes: motivación, difusión e insumos.

La motivación y difusión del programa RESA incluían una serie de actividades (reuniones con las comunidades y autoridades indígenas del resguardo Ticoya, plegables, programas de radio) que buscaban difundir la filosofía del programa, expresada en el lema "producir para no comprar lo que la tierra nos puede dar" (RESA, 2006). También se escogían los extensionistas del programa, constituido por muchachos de las comunidades que venían se obtener su diploma como 
técnicos forestales del Servicio Nacional de Aprendizaje, SENA. Se nombró uno por comunidad.

La primera actividad que realizó el proyecto con las comunidades beneficiarias incluyó la selección de familias que participarían del proyecto, la socialización del proyecto y la concertación sobre qué implementos requerían para mejorar sus sistemas productivos y por ende su seguridad alimentaria (RESA, 2006, p. 65).

Los insumos a su vez contenían dos subcomponentes: agrícola y pecuario. El primero incluía el suministro de herramientas (machete, pala, lima, hacha y rastrillo) y de semillas (cebolla, habichuela, lechuga, maíz, melón, pepino, sandía, tomate, cilantro, arroz, ahuyama, maracuyá y badea). En la programación inicial del programa RESA, se tenía contemplada la entrega de semillas de yuca y plátano (Red de Solidaridad, informe final RESA, 2006, p. 67). Pero ante la evidencia de lo absurdo que significaba entregar semillas que las comunidades beneficiarias poseían en abundancia, se decidió suministrar las semillas citadas. Esta situación pone de presente, una vez más, cómo RESA fue un programa concebido desde las oficinas del gobierno central y sin participación de las comunidades a las cuales se dirigió. En este subcomponente se realizaban, además, los talleres de capacitación en elaboración de abonos orgánicos, control ecológico de plagas, control fitosanitario y manejo de semillas.

El subcomponente pecuario comprendía la entrega de gallinas, gallos y malla metálica para la elaboración del gallinero. Y los talleres de capacitación en manejo de aves de corral, elaboración de alimento, enfermedades transmitidas por animales, medicina natural, preparación de alimentos y recetario amazónico.

Una vez entregados los materiales, la gente asumía que el programa había finalizado. Los talleres de capacitación se realizaron en forma irregular y lo principal, los talleres de elaboración de abonos orgánicos y control ecológico de plagas, no aprovecharon el conocimiento local. Los Ticuna, Cocama y Yagua, habitantes del resguardo Ticoya, poseen un elaborado conocimiento de la selva y sus recursos, que les ha permitido mantener por años sistemas productivos sostenibles. Este conocimiento incluye, por supuesto, la elaboración de abonos orgánicos y el control natural de plagas. 
El programa esperaba que la gente aplicara lo aprendido en los talleres de capacitación y elaborara el concentrado para alimentar las aves, pero en ninguna comunidad se hizo. El problema es que la gente no tuvo cómo alimentar las aves, una vez se terminó el bulto de concentrado que les entregaron al inicio del programa. Un miembro de la comunidad de Santa Teresita lo expresó claramente en los siguientes términos:

Yo digo eso, lo que el curaca dijo es cierto, porque ahorita sus gallinas está bueno porque tienes comida, pero después de que termina ese comida (concentrado de aves), ¿quién le mantiene ahí? donde se vaya muriendo de a uno a uno así que queda sin nada. Ahorita porque les está manteniendo con esa cosa (concentrado), pero después se van a aburrir, no les están dando nada. Toca sacar de vez en cuando para que la gallina conozca lo que va a comer, porque si mantiene encerrada, no va a conocer. Eso es, ahorita están bien sus gallinas pero después, hay algunos que no saben ni cuidar (Entrevista 03, comunidad de Santa Teresita, noviembre de 2009).

Un miembro de la comunidad Siete de Agosto es más explícito, y con mucho humor dijo lo siguiente:

Lo que no hemos podido ver hasta ahora es mantener la secuencia de eso, porque el proyecto dice, le damos hasta aquí de ahí pa'delante ya siguen ustedes, arranquen, pero como que llega hasta ahí, ya no hay seguimiento, empieza a venderse, se empieza a perderse, ya no hay productividad y ahí quedó, solo con el ejemplo de la gallina y lo que decía la abuela uitota algún día por ahí, decía dizque a la abuela gallina, muy bueno programa Estado, cuando tiene comida, cuando acaba comida gallina mirando a mí de hambre, queriendo comer a mí, antes que me coma a mí, yo como la gallina (risas) (Entrevista 02, comunidad de Siete de Agosto. noviembre de 2009).

Uno de los problemas generador de incomprensión y dudas entre el programa RESA y las comunidades beneficiarias, tuvo que ver con lo que la gente denomina la falta de pago o de un incentivo económico para que el programa pudiera funcionar y fuera acogido por las comunidades. 
Esta situación generó en las comunidades, lo que se denominó por parte de las instituciones responsables y de los extensionistas, falta de "interés” en el programa y fue la explicación del fracaso del programa RESA. Para despertar el interés en el programa se contrató, en cada comunidad, una persona que se desempeńó como extensionista. Los extensionistas fueron muchachos de las comunidades que recibieron su título de técnicos forestales del Servicio Nacional de Aprendizaje SENA. Su papel fue el de animar a las comunidades beneficiarias y hacer un seguimiento del programa. Para ello, recibieron un curso de capacitación ofrecido por las instituciones responsables del programa RESA, en especial del Instituto Sinchi.

El programa de desarrollo RESA, formó parte de un paquete tecnológico que exigió por parte de los beneficiarios, someterse a los rigores de la disciplina de trabajo, mediante la adopción de horarios laborales estrictos, con el fin de poner en marcha los proyectos productivos y garantizar su éxito. RESA asumió que la responsabilidad de los beneficiarios frente al programa se traducía en la cantidad de tiempo y jornadas de trabajo dedicadas a las labores del programa. A partir de su compromiso con el programa, los beneficiarios debían supeditar el tiempo que le dedican a sus demás labores productivas cotidianas en favor del programa de desarrollo. La pesca, las actividades propias de mantenimiento de la chagra tradicional, la recolección de productos del bosque maderables y no-maderables, la cacería e incluso la consecución de contratos de trabajo, debían relegarse al poco tiempo disponible después de cumplidas las exigentes y rutinarias labores del programa.

Es por esta razón que el programa de cría de aves, que forma parte de un paquete tecnológico establecido y definido de antemano mediante la utilización de la ciencia y la tecnología moderna, supone la construcción de un gallinero, la alimentación de las aves con concentrado y la aplicación de vacunas, pues al estar las aves reunidas en un gallinero, las posibilidad de adquirir enfermedades y parásitos como los piojos, es alta. Esta es, según el programa RESA, la forma adecuada de criar aves, pues de esta manera se garantiza un suministro regular de carne y huevos.

Los programas de desarrollo como RESA, están dirigidos a realzar la capacidad productiva del individuo y fomentar las responsabilidades individuales para el éxito 
del programa, en oposición a las formas colectivas de trabajo que practican los habitantes del resguardo Ticoya.

El conjunto de actividades que implica la crianza de aves en gallinero, exigiría a los beneficiarios de RESA, una dedicación casi exclusiva y un olvido de sus prácticas productivas que desempeñan cotidianamente, las cuales no son en sentido estricto, labores productivas, que comprenden únicamente aspectos económicos, de racionalidad económica, sino que estas se encuentran inmersas en un conjunto más amplio de relaciones sociales, sin las cuales los miembros de estas comunidades no pueden establecer y refrendar sus relaciones de solidaridad y reciprocidad. Estas relaciones sociales de solidaridad y reciprocidad a su vez se inscriben al interior de un sistema más amplio, las redes parentales. Estas últimas se refieren a los lazos de consanguinidad y afinidad establecidos entre las diferentes comunidades que conforman el resguardo Ticoya e incluso estas redes parentales se extienden a lo largo de la Amazonia central, entre las ciudades de Iquitos (Perú) y Manaos (Brasil).

$\mathrm{Al}$ respecto, una persona que se desempeñó como extensionista en la comunidad de Santa Teresita, en el río Loretoyacu, refirió su experiencia en los siguientes términos, en especial sobre la falta de interés en el programa RESA y la necesidad de que la gente reciba un pago a cambio de su trabajo:

Yo fui extensionista, a mí me pagaban una bonificación y nos capacitaron para seguir el proyecto, así se hicieron aquí algunas huertas, pero a la final no se puso cuidado y en la noche llegaron la arrieras y pin pin pin acabó con todo. Ahí terminó todo cuando llegó las arrieras, esa fue la segunda oportunidad. Eran semillas de cilantro, tomate, pimentones, sandías, pues terminó todo cuando llegaron las arrieras.

Cuando yo estuve de extensionista, eso fue lo que yo trataba de hacer con la gente: mire aquí hay unas semillas, estas semillas hay que sembrar y mucho no lo tomaron de importancia, algunos los guardaron y no hicieron el trabajo. Yo hice una muestra para ver si la gente la hacía, pero a muy poco les interesó, de lo que yo hice, alcancé a consumir, a sacar unos cilantro y pimentones, no me dio para más, porque las arrieras, tenía que tener mucho cuidado, para 
eso hay que tener el día completo, pero yo creo que donde hay una persona que se dedique solamente en la parte de agricultura, de tener esa garantía de estar ahí permanentemente lo logra tener todo eso, las verduras, las hortalizas que cultiva pero como uno a veces no le da tiempo a eso se pierde, pero uno poniéndole empeño, logra tener el cilantro en el caldito, que la fruta, todo eso. Yo le decía a la gente, porque cuando uno le enseñaba, ellos preguntaban que si para eso había plata, que ellos no lo iban a hacer gratis, entonces yo le decía que no era para eso, que era para mejorar la calidad de nutrición, la comida, que sea balanceada, yo les decía que no era solo el pescadito y la salsita, que al menos en un desayuno estuvieran diferentes nutrientes, la frutica, un jugo de papaya, eso le decía a la gente.

Cuando uno arranca, uno arranca con ganas, pero a los tiempos también la misma gente como no siguen el proceso, uno también se desanima, pero de todas maneras los proyectos son buenos, pero si el proyecto no viene y si no esté bien claro hacia la comunidad, la comunidad no lo va a hacer así, así usted le dé las herramientas, le dé todo, la comunidad no le va a poner la mano, la comunidad siempre quiere que usted le dé el incentivo (económico) para que se motiven más (Entrevista 03, comunidad de Santa Teresita, noviembre de 2009).

\section{Conclusiones}

El programa RESA, al igual que los demás programas de desarrollo, parten de una visión moderna del desarrollo, entendido como un discurso que dictamina una forma específica de entender la realidad y por lo tanto de determinar cuáles son las soluciones adecuadas que se plantean de acuerdo con la visión moderna del discurso del desarrollo.

Hasta los ańos cuarenta, se consideraba que los pueblos indígenas y en general los pueblos del Tercer Mundo, eran incapaces de asimilar la ciencia y la tecnología y por lo tanto era imposible que alcanzaran el progreso y el desarrollo económico.

Pero a partir del periodo de la posguerra, la pobreza, como lo señala Escobar (1998), se constituye en el punto de partida del discurso del desarrollo moderno. Para el desarrollo, 
la pobreza se relaciona con una serie de factores entrelazados entre sí, como la salud, la educación (entendida como la adquisición de valores modernos que permitirán la asimilación de la ciencia y la tecnología), la higiene y el ahorro entre otros, que en su conjunto se definirán como lo "social". La intervención en lo "social" se erige como el campo sobre el cual el discurso y el aparato institucional de desarrollo, van a centrar y desplegar sus acciones.

En ese sentido, RESA y los programas de desarrollo en general, entienden el principal problema de las comunidades indígenas y en este caso las comunidades beneficiarias, como un problema de pobreza. A partir de esta concepción, los programas de desarrollo están dirigidos a promover proyectos productivos que posibiliten tanto la producción de alimentos, como de ingresos económicos. Aunque estos últimos no forman parte de la filosofía de RESA, si constituye uno de los objetivos principales para las comunidades beneficiarias.

Un programa de desarrollo como RESA, parte del hecho de que la base técnica y científica que sustenta el programa, le imprime un carácter de éxito probado y verificable. Es por esto que asume que los beneficios que el programa aporta son evidentes, lo que permite que sea de fácil adopción por parte de los beneficiarios.

El paquete tecnológico de RESA supone la adopción de una estricta disciplina de trabajo, que olvida que una de las características de los pueblos indígenas amazónicos es la pluriactividad, lo que impide dedicarse exclusivamente a una sola actividad productiva. Es por esto que las acciones de capacitación no tuvieron el resultado esperado, pues ninguno de los participantes en las capacitaciones podía dedicarse exclusivamente a las actividades del programa RESA, pues pondría en riesgo la manutención de su familia y de su grupo doméstico, además de afectar su participación en actividades colectivas como las mingas, necesarias para mantener la red de relaciones en la cual él y su grupo doméstico se encuentran inmersos.

La racionalidad económica bajo la cual se inscriben los programas de desarrollo como RESA, impiden entender otras lógicas, que como las de los pueblos indígenas, no definen la organización de sus sociedades basados en las leyes del mercado y el crecimiento económico. Los programas de desarrollo como RESA, están dirigidos 
a realzar la capacidad productiva del individuo y fomentar las responsabilidades individuales para el éxito del programa, en oposición a las formas colectivas de trabajo que practican los habitantes del resguardo Ticoya.

\section{REFERENCIAS}

Acosta, L. E. y Mazorra, A. (2005). Enterramientos de masas de yuca del pueblo Ticuna: tecnología tradicional en la várzea del amazonas colombiano. Bogotá: Instituto Amazónico de Investigaciones Científicas.

Acosta, L. E., Peña, C. P. y Mazorra, A. (2006). Aportes del Programa RESA a la seguridad alimentaria de los pueblos indigenas del Amazonas. Pueblo Ticuna "Gente de huito y achiote", municipio de Puerto Nariño. Leticia, Amazonas: Instituto Amazónico de Investigaciones Científicas, SINCHI.

Dufour, D. L. (1990). Uso de la selva tropical por los indígenas Tukano del Vaupés. La selva humanizada: Ecología alternativa en el trópico húmedo colombiano. Bogotá, Fondo Editorial Cerec. pp. 43-58.

Escobar, A. (1998). La invención del Tercer Mundo. Construcción y deconstrucción del desarrollo. Bogotá: Grupo Editorial Norma.

Glenboski, L. L. (1977). Ethnobotany of the Tukuna Indians, Amazonas, Colombia, Ph. D. dissertation, University of Alabama.

Grenier, L. (1998). Connaissances indigènes et recherche. Un guide a l'intention des chercheurs. Ottawa, Canada: Centre de Recherches pour le développement International.

Gudeman, S. \& Rivera, A. (1990). Conversations in Colombia, the domestic economy in life and text. Cambridge: Cambridge University Press. 
Goulard, J. P. (2009). Entre Mortales e Inmortales. El Ser según los Ticuna de la Amazonia. Lima: Centro Amazónico de Antropología y Aplicación Práctica; Instituto Francés de Estudios Andinos.

León, A. M. (2009). Alimentación, nutrición y creencias alimentarias en San Martín de Amacayacu. Un estudio etnográfico. Tesis para la Maestría en Antropología: Universidad Nacional de Colombia.

Nimuendajú, C (1952). The Tukuna. Berkeley et Los Angeles: University of California Press.

Red de Solidaridad Social-Acción Social; Instituto De Investigaciones Científicas -SINCHI (2006). Informe final. Programa red de seguridad alimentaria RESA - RSS fortalecimiento del sistema de producción amazónico y mejoramiento de seguridad alimentaria de las comunidades indigenas del municipio y resguardo de puerto Nariño, departamento del Amazonas. Leticia, Colombia: Red de Solidaridad.

Trujillo, C. (2008). Selva y Mercado. Exploración cuantitativa de los ingresos en hogares indígenas. Tesis: Maestría en Estudios Amazónicos: Universidad Nacional de Colombia Sede Amazonia.

Universidad Javeriana (2007). Evaluación nacional del programa RESA, Acción Social, Presidencia de la república. Bogotá.

Viola, A. (2000). La crisis del desarrollismo y el surgimiento de la antropología del desarrollo. En: Viola, A. (Comp.) Antropología del desarrollo. Teoría y estudios etnográficos en América Latina. Barcelona, Ed.s. Paidós Ibérica S.A. pp. 9-64. 